Research Article

\title{
Consistent Conjectural Variations Equilibrium in the Semi-Mixed Oligopoly
}

\author{
Gabriela Renata Huarachi-Benavídez ${ }^{D},{ }^{1}$ José Guadalupe Flores-Muñiz ${ }^{(D)}{ }^{1}$ \\ Nataliya Kalashnykova, ${ }^{1}$ and Viacheslav Kalashnikov ${ }^{2}$ \\ ${ }^{1}$ Universidad Autónoma de Nuevo León (UANL), Av. Universidad S/N, Ciudad Universitaria, San Nicolás de los Garza, \\ Nuevo León 66455, Mexico \\ ${ }^{2}$ Instituto Tecnológico y de Estudios Superiores de Monterrey (ITESM), Campus Monterrey, Ave. Eugenio Garza Sada 2501 Sur, \\ Monterrey, Nuevo León 64849, Mexico
}

Correspondence should be addressed to José Guadalupe Flores-Muñiz; jfloresm@uanl.edu.mx

Received 15 March 2021; Revised 8 April 2021; Accepted 13 April 2021; Published 18 June 2021

Academic Editor: Mohammad Yazdi

Copyright (c) 2021 Gabriela Renata Huarachi-Benavídez et al. This is an open access article distributed under the Creative Commons Attribution License, which permits unrestricted use, distribution, and reproduction in any medium, provided the original work is properly cited.

\begin{abstract}
We study a variant of the mixed oligopoly model with conjectural variations equilibrium, in which one of the producers maximizes not his net profit but the convex combination of the latter with the domestic social surplus. The coefficient of this convex combination is named socialization level. The producers' conjectures concern the price variations depending upon their production output variations. In this work, we extend the models studied before, considering the case of the producers' cost functions being convex but not necessarily quadratic. The notion of exterior and interior equilibrium is introduced (similarly to previous works), developing a consistency criterion for the conjectures. Existence and uniqueness theorems are formulated and proven. Results concerning the comparison between conjectural variations, perfect competition, and Cournot equilibriums are provided. Based on these results, we formulate an optimality criterion for the election of the socialization level. The existence of the optimal socialization level is proven under the condition that the public company cannot be too weak as compared to the private firms.
\end{abstract}

\section{Introduction}

During the last 20 years, models of mixed oligopolies have become a very popular theme in the literature. The high interest in mixed oligopolies is due to their importance for the economies of Europe (Germany, England, and others), Canada, and Japan (see [1]). There are examples of mixed oligopolies in the United States such as the overnight delivery industries. Mixed oligopolies are also common in Eastern Europe and the former Soviet Union, where competition between public and private companies has existed and still exists in many industries such as banking, mortgage loans, life insurance, airlines, telecommunications, natural gas, electric power, railway, automotive, steel, education, hospitality, healthcare, broadcasting, and delivery services.
In contrast to the classical oligopoly, the mixed oligopoly usually boasts at least one special producer, apart from private producers maximizing his net profit. The special company usually deals with an objective function distinct from the net profit. Plenty of such models include a producer who maximizes domestic social surplus (see [1-4] and [5], to mention but a few). An income-per-worker function replaces the standard net profit objective function in some other papers (cf. [6-9]). Researchers $[10,11]$ examine the third kind of mixed oligopoly with an exclusive participant aiming to enhance a convex combination of his net profit and domestic social surplus.

In many of the abovementioned works, the authors study the mixed oligopoly models in the frameworks of Cournot, Hotelling, or Stackelberg. Notwithstanding, nowadays, a concept of conjectural variations equilibrium (CVE) 
introduced in $[12,13]$ as another possible solution form in static games is used ever wider. This concept puts that the producers behave as follows: each producer chooses his most favorable strategy having supposed that every adversary's action is a conjectural variation function of his own move.

In [14], a scheme different from $[12,13]$ is proposed for the concept of equilibrium with conjectural variations. In [14], it was assumed that every model's agent contemplates the variations of the total production volume as a response to his own output variations and thus evaluates his influence. The selection of the total output as the contemplated parameter is explained by the fact that the classical Cournot model was selected as the basic point. In more detail, instead of the classical Cournot hypothesis, it was supposed that every producer uses conjectural variations of the total market's volume in the function of the variation of its own production volume as follows:

$$
G_{i}(\eta)=G+\left(\eta-q_{i}\right) \omega_{i}\left(G, q_{i}\right)
$$

where

(i) $G$ is the total market production volume

(ii) $q_{i}$ is the quantity of the current production by producer $i$

(iii) $\eta$ is the expected production quantity by producer $i$

(iv) $G_{i}(\eta)$ is the total production volume conjectured by producer $i$ after his production volume changes from $q_{i}$ to $\eta$

The conjecture function $\omega_{i}\left(G, q_{i}\right)$ in formula (1) represents the influence coefficient of producer $i$. In the classical Cournot model, this coefficient is equal to 1 , and in the perfect competition model, it is equal to zero. For the given conjectures, under general enough assumptions, it was proved the existence and uniqueness of the abovementioned equilibrium. However, the following important question was not answered: Which conjectures can be considered as optimal ones?

To answer this important question, Bulavsky in [15] brought up a truly new approach. The first important variation proposed was in the structure of the demand. In the classical oligopoly models, the total volume of transactions drops when the price rises. However, there exists a dual postulate that the price increases as the demand grows. To eliminate this seeming contradiction, it is necessary to distinguish between two types of demand: the passive one and the active one. The standard demand function in the classical oligopoly models is used to describe the passive demand: the consumers wait, when a product is selling at a certain price, they decide whether to buy it or not. However, the active demand does not depend on the price and forms an additional component of the total demand. This component may reflect a rush demand, as well as a kind of demand related to some needs outside the model (for example, related to military actions).

The second important variation was the proposed procedure of verification for the consistency of the influence coefficients. Rather than assuming the equivalence (symmetry) of the producers in the oligopoly, it is supposed that every producer makes conjectures regarding, not the (optimal) response functions of each of the other producers, but only about the variations of the market clearing price depending upon (infinitesimal) variations in the same producer's output volume. Knowing the adversaries' conjectures (called influence coefficients), every producer smears on a verification procedure and reveals if his influence coefficient is consistent with those of the rest of the producers.

In the works [16-20], the results of [15] were extended to the mixed duopoly and oligopoly cases, respectively. In [18-20], partially mixed duopoly and oligopoly models were studied, i.e., where the semi-public company, the same as in $[12,13]$, maximizes the convex combination of the functions of social surplus and the participant's net utility function with a parameter $\beta \in(0,1]$, considering quadratic market cost functions of the market's producers. Furthermore, in the works mentioned above, it is shown that the consistent conjectural variations equilibrium (CCVE) and the classical Cournot-Nash equilibrium do not coincide, and in many applications, the CCVE models provide more efficient and attractive results than the classical models. In particular, when the CCVE concept is applied to the electricity market in [16], the consistent conjectural variations equilibrium led to better results for producers and consumers.

In Sections 2, 3, and 4 of the present study, we extend the results from $[18,19]$ to the case of convex (but not necessarily quadratic) cost functions. Based upon the established existence and uniqueness results for the conjectural variations equilibrium (called the exterior equilibrium) for any set of feasible conjectures, the notion of interior equilibrium is introduced by developing a consistency criterion for the conjectures (referred to as influence coefficients), and the existence theorem for the interior equilibrium (understood as the CVE with consistent conjectures) is proven.

When studying oligopolies in addition to the problems of existence and calculation of the equilibrium states, much importance and interest is attracted by the comparative analysis of the different equilibria. Such a comparative analysis for the semi-mixed duopoly has been realized in [21]. Based on this analysis, in [21], we formulated the optimality criterion for the socialization level $\beta$ and proved the existence of the optimal socialization level $\beta^{\text {opt }} \in(0,1)$. In Section 5 of the present study, this analysis is extended to the oligopoly case, where all private firms have the same cost function, the optimality criterion for the election of the optimal socialization level has been formulated, and its existence is demonstrated.

In Section 6, we describe the methodology and algorithms used to find the equilibriums and the optimal value of $\beta$. In Section 7 , some numerical experiments, together with the analysis of the results, are provided. Concluding remarks (Section 8), funding bodies, and the list of references complete the paper. The proofs of the lemmas and theorems can be found in the Supplementary Materials. 


\section{The Model's Specification}

Let us consider an oligopoly market of one homogeneous product with $n+1$ producers, $n \geq 1$. Each producer $i \in\{0,1, \ldots, n\}$ has its cost function $f_{i}\left(q_{i}\right)$, where $q_{i}$ is the production volume for every producer $i$.

In the classical oligopoly models, the total volume of products in the market usually decreases as the price rises. The latter agrees with the postulate that the price falls when the supply increases.

However, there is a second postulate: the price rises with an increase in demand. To deal with this apparent contradiction, we need to distinguish between 2 types of demand: passive and active.

The passive demand in the oligopoly models is a function that describes the behavior of the consumers who take into account the price of a product to decide whether to buy it or not.

On the other hand, the active demand reflects the behavior of the consumers who buy the product regardless of the price. Thus, in theory, an increment in the active demand should raise the price in the market. This component can be represented by an urgent demand or the demand associated with some needs outside the model, for example, related to military operations, the development of defense industries, or something else. The possible dependence of the active demand upon the price in the market is outside the studied model.

Therefore, we consider here both types of demand. The passive demand depends on the price and is determined by the function $G(p)$, where $p$ is the market price proposed by all producers. The active demand $D$ is nonnegative and does not depend on the market price.
The equilibrium between the offer and the demand, for the price value $p$, is defined by the following balance equation:

$$
\sum_{i=0}^{n} q_{i}=G(p)+D
$$

The behavior of the passive demand described above is represented in the following assumption:

A1. The demand function $G(p)$ is defined for price values $p \in(0,+\infty)$, is nonnegative, continuously differentiable, and $G^{\prime}(p)<0$.

Moreover, since we consider total cost functions, we assume that these functions are strictly increasing and strictly convex. This is described by the following assumption:

A2. For each producer $i \in\{0,1, \ldots, n\}$, its cost function, $f_{i}\left(q_{i}\right)$, is defined for each $q_{i} \geq 0$ and is twice continuously differentiable with $f_{i}^{\prime}(0)>0$ and $f_{i}^{\prime \prime}\left(q_{i}\right)>0$. In addition,

$$
f_{0}^{\prime}(0) \leq \max _{i \in\{1, \ldots, n\}}\left\{f_{i}^{\prime}(0)\right\} .
$$

The producers $i \in\{1, \ldots, n\}$ are called private producers and they select their production volume $q_{i}$ in order to maximize its net profit function:

$$
\pi_{i}\left(p, q_{i}\right)=p q_{i}-f_{i}\left(q_{i}\right) .
$$

On the other hand, producer $i=0$ is a semi-public company and it selects its production volume $q_{0}$ such that it maximizes the convex combination of the functions of the social surplus and the net utility function:

$$
\begin{aligned}
S\left(\beta, p, q_{0}, q_{1}, \ldots, q_{n}\right)= & \beta\left(\int_{0}^{\sum_{i=0}^{n} q_{i}} p(x) \mathrm{d} x-p \sum_{i=1}^{n} q_{i}-f_{0}\left(q_{0}\right)\right) \\
& +(1-\beta)\left(p q_{0}-f_{0}\left(q_{0}\right)\right),
\end{aligned}
$$

where, like in $[10,11], \beta \in(0,1]$ is a parameter. From now on, we will call this parameter, $\beta$, socialization level.

Assume that all the producers (both semi-public and private) accept that the election of their production volumes will affect the market price value $p$. In this way, the firstorder optimality conditions describing the market equilibrium will have the following form:
For private producers $(i \in\{1, \ldots, n\})$,

$$
\frac{\partial \pi_{i}}{\partial q_{i}}=p+q_{i} \frac{\partial p}{\partial q_{i}}-f_{i}^{\prime}\left(q_{i}\right) \begin{cases}=0, & \text { if } q_{i}>0 \\ \leq 0, & \text { if } q_{i}=0 .\end{cases}
$$

And for the semi-public producer $(i=0)$,

$$
\frac{\partial S}{\partial q_{0}}=p+\left[(1-\beta) q_{0}-\beta \sum_{i=1}^{n} q_{i}\right] \frac{\partial p}{\partial q_{0}}-f_{0}^{\prime}\left(q_{0}\right) \begin{cases}=0, & \text { if } q_{0}>0 \\ \leq 0, & \text { if } q_{0}=0 .\end{cases}
$$


As we can see, in order to describe the behavior of the producers, the most important is to know, not the function $p$, but rather its derivatives:

$$
\frac{\partial p}{\partial q_{i}}=-v_{i}
$$

Here, we introduce the negative sign in (8) in order to work with nonnegative values of $v_{i}$.

In addition, we must guarantee that the derivatives of $p$ with respect to its production volume $i \in\{0,1, \ldots, n\}$ provide the concavity of the function. Otherwise, we cannot guarantee that the necessary conditions are also sufficient.

For private producers $i \in\{1, \ldots, n\}$ in order for conditions (6) to be sufficient, one has to guarantee that the function $\pi_{i}\left(q_{i}\right)$ is concave. As we suppose that the cost functions are strictly convex, it lacks only the concavity of the product $p q_{i}$, with respect to $q_{i}$, to guarantee that. But for the latter, it is sufficient to suppose that the coefficient $v_{i}$ (which we call influence coefficient of producer $i$ ) is nonnegative and constant. In the latter case, the function of the conjectured local dependence for the net utility of the private producer $i$ with respect to the variation of his production volume $\eta_{i}$ has the form

$$
\widehat{\pi}_{i}\left(\eta_{i}\right)=\left[p-v_{i}\left(\eta_{i}-q_{i}\right)\right] \eta_{i}-f_{i}\left(\eta_{i}\right),
$$

which is a quadratic and concave function.

Therefore, the first-order necessary (and now sufficient) conditions for the equilibrium's production volume, $\eta_{i}=q_{i}$, to be optimal are given as follows:

$$
\left\{\begin{array}{ll}
p=v_{i} q_{i}+f_{i}^{\prime}\left(q_{i}\right), & \text { if } q_{i}>0, \\
p \leq f_{i}^{\prime}(0), & \text { if } q_{i}=0,
\end{array} \quad i \in\{1, \ldots, n\} .\right.
$$

In a similar manner, the semi-public producer's conjectured local dependence of its objective function has the form

$$
\widehat{S}\left(\eta_{0}\right)=\beta\left(\int_{0}^{\eta_{0}+\sum_{i=1}^{n} q_{i}} p(x) \mathrm{d} x-\left[p-\nu_{0}\left(\eta_{0}-q_{0}\right)\right] \sum_{i=1}^{n} q_{i}-f_{0}\left(\eta_{0}\right)\right)+(1-\beta)\left(\left[p-\nu_{0}\left(\eta_{0}-q_{0}\right)\right] \eta_{0}-f_{0}\left(\eta_{0}\right)\right)
$$

which is also a concave function and allows one to obtain the necessary and sufficient conditions for the equilibrium production volume, $\eta_{0}=q_{0}$, to be optimal, which are as follows:

$$
\begin{cases}p=\left[(1-\beta) q_{0}-\beta \sum_{i=1}^{n} q_{i}\right] \nu_{0}+f_{0}^{\prime}\left(q_{0}\right), & \text { if } q_{0}>0, \\ p \leq-\beta\left(\sum_{i=1}^{n} q_{i}\right) \nu_{0}+f_{0}^{\prime}(0), & \text { if } q_{0}=0 .\end{cases}
$$

If the producers' conjectures are given exogenously, then its production volumes are determined uniquely as a function of the model's parameters and also of the other producers' volumes. This equilibrium is called exterior and is described by the vector $\left(p, q_{0}, q_{1}, \ldots, q_{n}\right)$.

However, in this work, we study the second concept of equilibrium, called interior (consistent), in which the influence coefficients $v_{i}$ are not given beforehand but are found from the models' data by solving a special system of equations.

\section{Exterior Equilibrium}

Definition 1 . The vector $\left(p, q_{0}, q_{1}, \ldots, q_{n}\right)$ is called the exterior equilibrium for the influence coefficients $\left(v_{0}, v_{1}, \ldots, v_{n}\right), v_{i} \geq 0, i \in\{0,1, \ldots, n\}$, whenever the balance equation (2) is valid, and for all the producers, the optimality conditions (10) and (12) are valid.
From now on, we assume that the number of participants in the model is always the same regardless of the influence coefficients. In order to guarantee that, we introduce the value $p_{0}$ and prove Lemma 1 presented below:

$$
p_{0}=\max _{i \in\{1, \ldots, n\}}\left\{f_{i}^{\prime}(0)\right\} \geq f_{0}^{\prime}(0) .
$$

Lemma 1. Let assumptions $A 1$ and $A 2$ be true. If the vector $\left(p, q_{0}, q_{1}, \ldots, q_{n}\right)$ is the exterior equilibrium for the given influence coefficients $\left(v_{0}, v_{1}, \ldots, v_{n}\right)$, then the relation $p>p_{0}$ is equivalent to the fact that all production volumes are positive, i.e., $q_{i}>0, \forall i \in\{0,1, \ldots, n\}$.

Now, we introduce the following assumption:

$A 3$. For the price $p_{0}$ and for any $v_{i} \geq 0, i \in\{0,1, \ldots, n\}$, there exists the production volume $q_{i}^{0} \geq 0$ (unique by A2), such that

$$
p_{0}=f_{i}^{\prime}\left(q_{i}^{0}\right)
$$

and in addition,

$$
\sum_{i=0}^{n} q_{i}^{0}<G\left(p_{0}\right)
$$

Assumption A3, together with A1 and A2, guarantees the existence and uniqueness of the exterior equilibrium for any nonnegative values of $v_{i} \geq 0, i \in\{1, \ldots, n\}$, and $v_{0} \in\left[0, \bar{v}_{0}\right)$, where 


$$
\bar{\nu}_{0}= \begin{cases}\frac{f_{0}^{\prime}\left(G\left(p_{0}\right)-\sum_{i=1}^{n} q_{i}^{0}\right)-p_{0}}{\sum_{i=1}^{n} q_{i}^{0}}, & \text { if } \beta=1, \sum_{i=1}^{n} q_{i}^{0}>0, \\ \frac{f_{0}^{\prime}\left(G\left(p_{0}\right)-\sum_{i=1}^{n} q_{i}^{0}\right)-p_{0}}{\sum_{i=1}^{n} q_{i}^{0}-(1-\beta) G\left(p_{0}\right)}, & \text { if } \beta \in(0,1), \sum_{i=1}^{n} q_{i}^{0}>\max \left\{(1-\beta) G\left(p_{0}\right), \frac{1-\beta}{\beta} q_{0}^{0}\right\}, \\ +\infty, & \text { otherwise. }\end{cases}
$$

This fact is formulated and established in the following theorem.

Theorem 1. Under assumptions $A 1, A 2$, and $A 3$, for any $\beta \in(0,1], D \geq 0, v_{i} \geq 0, i \in\{1, \ldots, n\}$, and $v_{0} \in\left[0, \bar{v}_{0}\right)$, there exists uniquely the exterior equilibrium $\left(p, q_{0}, q_{1}, \ldots, q_{n}\right)$, which is continuously differentiable with respect to the parameters $\beta, D$, and $v_{i}, i \in\{0,1, \ldots, n\}$. Moreover, $p>p_{0}$ and

$$
\frac{\partial p}{\partial D}=\frac{1}{\left(1 /\left((1-\beta) v_{0}+f_{0}^{\prime \prime}\left(q_{0}\right)\right)\right)+\left(\left(v_{0}+f_{0}^{\prime \prime}\left(q_{0}\right)\right) /\left((1-\beta) v_{0}+f_{0}^{\prime \prime}\left(q_{0}\right)\right)\right) \sum_{i=1}^{n}\left(1 /\left(v_{i}+f_{i}^{\prime \prime}\left(q_{i}\right)\right)\right)-G^{\prime}(p)} .
$$

\section{Interior Equilibrium}

Before defining the key concept of interior equilibrium, we recall the procedure of verification of the equilibrium introduced in [22]. Suppose that, for some parameters $\left(v_{0}, v_{1}, \ldots, v_{n}\right)$ and $D$, the exterior equilibrium $\left(p, q_{0}, q_{1}, \ldots, q_{n}\right)$ has been found. Now assume that one of the producers, for example, producer $k$, temporarily changes his behavior and stops maximizing his expected net profit and starts making small variations around his optimal production volume $q_{k}$. The latter is equivalent to considering that only the producers belonging to the set $I_{-k}=\{0,1, \ldots, n\} \backslash\{k\}$ continue to operate in the model, subtracting the production volume $q_{k}$ from the active demand $D$. In this case, the variations of the production volume $q_{k}$ (from producer $k$ ) are equivalent to the variations (in the opposite direction) of the active demand $D_{k}=D-q_{k}$. Considering the variations of $q_{k}$ as infinitesimal, we can suppose that, by observing the variations in the equilibrium price $p$ corresponding to the variations of the active demand $D_{k}$, producer $k$ can obtain the variations in $p$ with respect to his production volume $q_{k}$, given in the form

$$
\frac{\partial p}{\partial D_{k}}=\frac{\partial p}{\partial\left(D-q_{k}\right)}=-\frac{\partial p}{\partial q_{k}}=v_{k}
$$

which is his influence coefficient.

In the proposed model, if producer $k \in\{1, \ldots, n\}$ leaves the market, in order to calculate the corresponding derivative $\partial p / \partial D_{k}$ we apply formula (17) from Theorem 1 , remembering that producer $k$ is absent, so the terms corresponding to the index $i=k$ must be excluded from the formula. On the other hand, if the semi-public producer $i=$ 0 is the one who leaves the market, then only private producers will remain in the model; thus, we have to apply the theorem and the formula obtained in [15] for the classical oligopoly model. With this in mind, we obtain the following criterion.

Definition 2 (consistency criterion). We say that the influence coefficients $\left(v_{0}, v_{1}, \ldots, v_{n}\right)$ are consistent if they, together with their corresponding exterior equilibrium $\left(p, q_{0}, q_{1}, \ldots, q_{n}\right)$, satisfy the following equalities:

$$
v_{0}=\frac{1}{\sum_{i=1}^{n}\left(1 /\left(\nu_{i}+f_{i}^{\prime \prime}\left(q_{i}\right)\right)\right)-G^{\prime}(p)},
$$

and for all $i \in\{1, \ldots, n\}$,

$$
v_{i}=\frac{1}{\left(1 /\left((1-\beta) v_{0}+f_{0}^{\prime \prime}\left(q_{0}\right)\right)\right)+\left(\left(v_{0}+f_{0}^{\prime \prime}\left(q_{0}\right)\right) /\left((1-\beta) v_{0}+f_{0}^{\prime \prime}\left(q_{0}\right)\right)\right) \sum_{\substack{j=1 \\ j \neq i}}^{n}\left(1 /\left(v_{j}+f_{j}^{\prime \prime}\left(q_{j}\right)\right)\right)-G^{\prime}(p)} .
$$

Now we can define the concept of interior equilibrium.

Definition 3. The collection $\left(p, q_{0}, q_{1}, \ldots, q_{n}, v_{0}, v_{1}, \ldots, v_{n}\right)$ where $v_{i} \geq 0, i \in\{0,1, \ldots, n\}$, is called the interior equilibrium if, for the given influence coefficients $\left(v_{0}, v_{1}, \ldots, v_{n}\right)$, the vector $\left(p, q_{0}, q_{1}, \ldots, q_{n}\right)$ is the exterior equilibrium, and the consistency criterion (Definition 2) is valid, i.e., equations (19) and (20) are satisfied for all $i \in\{0,1, \ldots, n\}$.

The existence of the interior equilibrium has been formulated and proven in the following theorem. 
Theorem 2. Under assumptions A1, A2, and A3, there exists the interior equilibrium.

\section{A Particular Case: Affine Demand Function, Identical Private Producers, and Quadratic Cost Functions}

When studying an oligopoly in the framework proposed in this study, apart from the questions of the existence of the consistent equilibrium and how to calculate it, special attention is paid to the comparison of the latter with the ones appearing in the Cournot model and the perfect competition model.

Let us consider a particular case in which the active demand is zero, the passive demand is a piecewise linear function, all the producers have a quadratic cost function, and this cost function is the same for every private producer.

For this particular case, we reformulate the main model's assumptions, $\mathrm{A} 1$ and $\mathrm{A} 2$, as follows:

A4. The passive demand is given by the piecewise function

$$
G(p)= \begin{cases}-K p+T, & \text { if } 0<p<\frac{T}{K}, \\ 0, & \text { if } p \geq \frac{T}{K},\end{cases}
$$

where $K>0$ and $T>0$.

A5. The cost functions are quadratic, i.e.,

$$
f_{0}\left(q_{0}\right)=\frac{1}{2} a_{0} q_{0}^{2}+b_{0} q_{0}, \quad a_{0}>0, b_{0}>0,
$$

and for all $i \in\{1, \ldots, n\}$

$$
f_{i}\left(q_{i}\right) \equiv f(q)=\frac{1}{2} a q^{2}+b q, \quad a>0, b \geq b_{0} .
$$

In this particular case, since the demand function $G(p)$ is piecewise linear, it is nondifferentiable at the point $p=T / K$, so assumption A1 does not hold. However, from the proof of Theorem 1, it follows that the price $p$ of the exterior equilibrium $\left(p, q_{0}, q_{1}, \ldots, q_{n}\right)$ is given by the intersection of the total volume function $Q(p)=\sum_{i=0}^{n} q_{i}(p)$ and the demand function $G(p)$; moreover, this intersection lies within the open interval $\left(p_{0}, T / K\right)$ where the demand function defined in assumption A4 is given by $G(p)=-K p+T>0$, thus satisfying the conditions of assumption A1. Thus, the results presented in the previous section hold true for this particular case.

Assumption $\mathrm{A} 3$ is reformulated in the following form:

$A 6$. For the price $p_{0}=b$, it holds that

$$
\frac{p_{0}-b_{0}}{a_{0}}<-K p_{0}+T
$$

The balance equality takes the form

$$
q_{0}+n q=-K p+T .
$$

The net utility function for private producers is reduced to

$$
\pi(p, q)=p q-\left(\frac{1}{2} a q^{2}+b q\right) .
$$

However, the objective function for the semi-public producer transforms into

$$
\begin{aligned}
S\left(\beta, p, q_{0}, q\right)= & \beta\left[\int_{0}^{q 0+n q} p(x) \mathrm{d} x-n p q-\left(\frac{1}{2} a_{0} q_{0}^{2}+b_{0} q_{0}\right)\right] \\
& +(1-\beta)\left[p q_{0}-\left(\frac{1}{2} a_{0} q_{0}^{2}+b_{0} q_{0}\right)\right] .
\end{aligned}
$$

The first-order necessary (and sufficient) optimality conditions are as follows.

For the private producers,

$$
\begin{cases}p=v q+a q+b, & \text { if } q>0 \\ p \leq b, & \text { if } q=0\end{cases}
$$

And for the semi-public producer,

$$
\begin{cases}p=\left[(1-\beta) q_{0}-n \beta q\right] v_{0}+a q_{0}+b_{0}, & \text { if } q_{0}>0, \\ p \leq-n \beta q v_{0}+b_{0}, & \text { if } q_{0}=0 .\end{cases}
$$

5.1. Analysis of the Consistent Equilibrium. Taking into account that in the considered particular case, all private producers have the same influence coefficient, i.e., $v_{i} \equiv v, \forall i \in\{1, \ldots, n\}$, we obtain the following consistency criterion.

Definition 4 (consistency criterion for the particular case). The influence coefficients $\left(\nu_{0}, \nu\right)$ are consistent for the corresponding exterior equilibrium $\left(p, q_{0}, q\right)$, if the following equalities are valid:

$v_{0}=\frac{1}{(n /(v+a))+K}$,

$$
\nu=\frac{1}{\left(1 /\left((1-\beta) v_{0}+a_{0}\right)\right)+\left(\left(v_{0}+a_{0}\right) /\left((1-\beta) v_{0}+a_{0}\right)\right)((n-1) /(v+a))+K} .
$$

Theorem 3. Under assumptions A4, A5, and A6, for every $\beta \in(0,1]$, there exists uniquely the interior equilibrium $\left(p^{*}, q_{0}^{*}, q^{*}, v_{0}^{*}, v^{*}\right)$.

By Theorem 3, for each $\beta \in(0,1]$, there exists uniquely the consistent (interior) equilibrium $\left(p^{*}(\beta), q_{0}^{*}(\beta), q^{*}(\beta)\right.$, $\left.v_{0}^{*}(\beta), v^{*}(\beta)\right)$, and we denote the profit of private producers calculated for this equilibrium by $\pi^{*}(\beta)=\pi\left(p^{*}(\beta), q^{*}(\beta)\right)$. 
Theorem 4. The interior equilibrium $\left(p^{*}(\beta), q_{0}^{*}(\beta), q^{*}(\beta)\right.$, $\left.v_{0}^{*}(\beta), v^{*}(\beta)\right)$ and the function $\pi^{*}(\beta)$ are continuously differentiable with respect to $\beta \in(0,1]$. Moreover, the functions $p^{*}(\beta), v_{0}^{*}(\beta)$, and $\nu^{*}(\beta)$ are strictly decreasing for all $\beta \in(0,1]$.

5.2. Analysis of the Cournot Equilibrium. The Cournot conjecture for oligopoly models is described by the following identity:

$$
\omega_{i}=\frac{\partial G}{\partial q_{i}}=1, \quad \forall i \in\{0,1, \ldots, n\} .
$$

The previous identity (32), in our model, corresponds to the following equality for the influence coefficients:

$$
v_{i}=-\frac{\partial p}{\partial q_{i}}=-\frac{\omega_{i}}{G^{\prime}(p)}=-\frac{1}{G^{\prime}(p)}, \quad \forall i \in\{0,1, \ldots, n\} .
$$

Therefore, for our particular case, the Cournot conjecture is given by

$$
\nu_{0}=v=\frac{1}{K}
$$

By Theorem 1 , for every $\beta \in(0,1]$, there exists uniquely the exterior equilibrium $\left(p^{c}(\beta), q_{0}^{c}(\beta), q^{c}(\beta)\right)$, corresponding to the Cournot conjecture, which we denote for our particular case by $\nu_{0}^{c}=\nu^{c}=1 / K$.

It is easy to see that the Cournot equilibrium does not coincide with the interior (consistent) equilibrium in our model because the consistency criterion is not satisfied. Indeed,

$$
\begin{aligned}
& \frac{1}{\left(n /\left(\nu^{c}+a\right)\right)+K}<\frac{1}{K}=v_{0}^{c}, \\
& \frac{1}{\left(1 /\left((1-\beta) \nu_{0}^{c}+a_{0}\right)\right)+\left(\left(v_{0}^{c}+a_{0}\right) /\left((1-\beta) \nu_{0}^{c}+a_{0}\right)\right)\left((n-1) /\left(\nu^{c}+a\right)\right)+K}<\frac{1}{K}=v^{c} .
\end{aligned}
$$

For each $\beta \in(0,1]$, we denote the net profit of private producers for the Cournot equilibrium by $\pi^{c}(\beta)=\pi\left(p^{c}(\beta), q^{c}(\beta)\right)$.

Theorem 5. Under assumptions A4, A5, and A6, the exterior equilibrium $\left(p^{c}(\beta), q_{0}^{c}(\beta), q^{c}(\beta)\right)$ and the function $\pi^{c}(\beta)$ are continuously differentiable with respect to $\beta \in(0,1]$. Moreover, the function $p^{c}(\beta)$ is strictly decreasing for all $\beta \in(0,1]$.

5.3. Analysis of the Perfect Competition Equilibrium. The perfect competition conjecture for oligopoly models is represented by the following identities:

$$
\omega_{i}=\frac{\partial G}{\partial q_{i}}=0, \quad \forall i \in\{0,1, \ldots, n\},
$$

which in our model corresponds to the following influence coefficients:

$$
v_{i}=-\frac{\partial p}{\partial q_{i}}=-\frac{\omega_{i}}{G^{\prime}(p)}=0, \quad \forall i \in\{0,1, \ldots, n\} .
$$

The latter, for our particular case, is reduced to

$$
v_{0}=v=0 \text {. }
$$

By Theorem 1 , for each $\beta \in(0,1]$, there exists uniquely the exterior equilibrium $\left(p^{t}(\beta), q_{0}^{t}(\beta), q^{t}(\beta)\right)$, corresponding to the perfect competition conjecture, which is denoted in our particular case by $\nu_{0}^{t}=v^{t}=0$.

It is easy to check that the perfect competition equilibrium does not match with the consistent equilibrium in our model since the consistency criterion is not met. Indeed,

$$
\begin{aligned}
& \frac{1}{\left(n /\left(\nu^{t}+a\right)\right)+K}>0=v_{0}^{t}, \\
& \frac{1}{\left(1 /\left((1-\beta) \nu_{0}^{t}+a_{0}\right)\right)+\left(\left(v_{0}^{t}+a_{0}\right) /\left((1-\beta) \nu_{0}^{t}+a_{0}\right)\right)\left((n-1) /\left(\nu^{t}+a\right)\right)+K}>0=v^{t} .
\end{aligned}
$$

For each $\beta \in(0,1]$, we denote the net profit of private producers for the perfect competition equilibrium by $\pi^{t}(\beta)=\pi\left(p^{t}(\beta), q^{t}(\beta)\right)$.
Theorem 6. Under assumptions $A 4, A 5$, and $A 6$, the exterior equilibrium $\left(p^{t}(\beta), q_{0}^{t}(\beta), q^{t}(\beta)\right)$ and the function $\pi^{t}(\beta)$ are constant with respect to $\beta \in(0,1]$. 
5.4. Comparative Analysis. It is of great interest the comparison among the three types of equilibrium: the consistent (interior) equilibrium, the Cournot equilibrium, and the perfect competition equilibrium. For the case of duopoly, i.e., when $n=1$, the analysis of these three equilibria can be found in [21]. Because of that, in this study, we consider only the case of oligopoly, i.e., when $n \geq 2$.

Theorem 7. Under assumptions A4, A5, and A6, the following inequalities hold:

$$
\lim _{\beta \downarrow 0} p^{c}(\beta)>\lim _{\beta \downarrow 0} p^{*}(\beta)>p^{t} .
$$

For the case of duopoly, it was shown in [21] that for any $\beta \in(0,1]$, the inequality $p^{c}(\beta)>p^{*}(\beta)$ holds. However, for the case of oligopoly in our model, inequality (40) between the prices when $\beta \uparrow 1$ may or may not hold, depending on the model's parameters. Examples of both situations can be found in the numerical experiments of Section 7.

Theorem 8. Under assumptions A4, A5, and A6, for any $\beta \in(0,1]$, if $\pi^{c}(\beta) \geq \pi^{*}(\beta)$, then it is satisfied that $p^{*}(\beta)<p^{c}(\beta)$.

Theorem 9. Suppose that assumptions A4, A5, and A6 are true. If the relationship

$$
\frac{(n-1) a}{n+a K} \geq a_{0}
$$

is valid, then there exists the value $\widehat{\beta} \in(0,1)$ such that $\pi^{c}(\widehat{\beta})=\pi^{*}(\widehat{\beta})$ and $p^{*}(\widehat{\beta})<p^{c}(\widehat{\beta})$.

In other words, if relationship (41) holds, we may consider this value $\widehat{\beta}$ (from Theorem 9 ) as the optimal socialization level. Indeed, for this value $\widehat{\beta}$, the net profits for the private producers are the same in both, the Cournot equilibrium and the consistent equilibrium; hence, the semipublic company can convince the private firms to change their Cournot strategies for the consistent strategies, guaranteeing that the market price will be lower than the one appearing in the Cournot model. Thereby, the semi-public company is not only fulfilling its social responsibility, but also will keep safe its own budget since it does not need to pay any subsidies to the private firms nor the consumers.

The existence of this optimal socialization level was shown in [21] for the case of a partially mixed duopoly without the need of relation (41), but in the oligopoly case, this optimal socialization level may not exist (indeed, it might be that $\pi^{*}(\beta)<\pi^{c}(\beta)$ for all $\left.\beta \in(0,1]\right)$; the latter can happen when the semi-public company is much weaker than the private firms. For such cases, we plan (in future works) to make use of the politics of subsidies in order to define a different optimality criterion for the socialization level.

\section{Solution Methodology}

In this section, we describe the methodology and algorithms used to find the exterior equilibrium, the interior equilibrium, and the optimal socialization level for the particular case introduced in Section 5.

To find the exterior equilibrium $\left(p^{*}, q_{0}^{*}, q^{*}\right)$ for a fixed set of values $\beta \in(0,1]$ and $\nu_{0}, \nu \geq 0$, we first isolate the variables $q$ and $q_{0}$ from the first-order necessary conditions (28) and (29), assuming that $q_{0}, q>0$. Thus, we define the continuous functions $q(p, v)$ and $q_{0}\left(p, \beta, v_{0}, v\right)$, given by the equations (E.25) and (E.26), respectively, in Supplementary Materials, respectively, within the proof of Theorem 4.

Now, by assumption $\mathrm{A} 6$, for the price $p_{0}=b$, we have that

$$
q_{0}\left(p_{0}, \beta, v_{0}, v\right)+q\left(p_{0}, v\right)<G\left(p_{0}\right)
$$

and from Theorem 3, we know that there exists uniquely the exterior equilibrium, and thus, we can find a value $p_{1}>p_{0}$ large enough (since the functions $q_{0}$ and $q$ are strictly increasing with respect to $p$, and $G$ is strictly decreasing with respect to $p$ ) such that

$$
q_{0}\left(p_{1}, \beta, v_{0}, v\right)+q\left(p_{1}, v\right)>G\left(p_{1}\right)
$$

After finding the value $p_{1}$, the exterior equilibrium's price $p^{*}$ is given by the intersection of the functions

$$
\begin{array}{r}
q_{0}\left(p, \beta, \nu_{0}, \nu\right)+q(p, \nu), \\
G(p),
\end{array}
$$

in the variable $p$ within the interval $\left(p_{0}, p_{1}\right)$, which we can easily find using a bisection algorithm.

Finally, the exterior equilibrium's volume outputs are given by the values $q_{0}^{*}=q_{0}\left(p^{*}, \beta, v_{0}, v\right)$ and $q^{*}=q\left(p^{*}, v\right)$.

To find the interior equilibrium $\left(p^{*}, q_{0}^{*}, q^{*}, v_{0}^{*}, v^{*}\right)$ for a fixed value $\beta \in(0,1]$, we first define a map with the righthand sides of the consistency criterion's equations (30) and (31). The defined map $H\left(\nu_{0}, \nu\right)=\left(F_{0}\left(\nu_{0}, \nu\right), F\left(\nu_{0}, \nu\right)\right)$ is given by the functions (D.3) and (D.4) in Supplementary Materials within the proof of Theorem 3.

From the proof of Theorem 3, we also know that the map $H\left(\nu_{0}, \nu\right)$ is a contraction and has a unique fixed point in the region $v_{0}, v \geq 0$. This fixed point is the vector of consistent conjectures $\left(v_{0}^{*}, v^{*}\right)$, which we can find by applying the iterated function sequence

$$
\left(v_{0}^{k+1}, v^{k+1}\right)=H\left(v_{0}^{k}, v^{k}\right)
$$

starting with the point $\left(\nu_{0}^{0}, v^{0}\right)=(0,0)$, until the distance between $\left(v_{0}^{k}, v^{k}\right)$ and $H\left(v_{0}^{k}, v^{k}\right)$ is small enough.

Finally, the values of $\left(p^{*}, q_{0}^{*}, q^{*}\right)$ are given by the exterior equilibrium corresponding to $\left(v_{0}^{*}, v^{*}\right)$.

To find the optimal socialization level $\widehat{\beta}$, we first define the private firms' profit functions $\pi^{*}(\beta)=\pi\left(p^{*}(\beta), q^{*}(\beta)\right)$ and $\pi^{c}(\beta)=\pi\left(p^{c}(\beta), q^{c}(\beta)\right)$, for the consistent and Cournot equilibriums, respectively, in the variable $\beta \in(0,1]$, where $\pi(p, q)$ is the net profit for the private firms given by (26), the vector $\left(p^{*}(\beta), q_{0}^{*}(\beta), q^{*}(\beta), v_{0}^{*}(\beta), v^{*}(\beta)\right)$ is the interior equilibrium for the value $\beta$, and the vector $\left(p^{c}(\beta), q_{0}^{c}(\beta), q^{c}(\beta)\right)$ is the exterior equilibrium for the value $\beta$ and the influence coefficients $\left(\nu_{0}^{c}, v^{c}\right)=(1 / K, 1 / K)$.

From the proof of Theorem 9, we know that 


$$
\lim _{\beta \downarrow 0} \pi^{*}(\beta)<\lim _{\beta \downarrow 0} \pi^{c}(\beta),
$$

so we can find a small enough $\beta_{0} \in(0,1)$ such that $\pi^{*}\left(\beta_{0}\right)<\pi^{c}\left(\beta_{0}\right)$. Also from the proof of Theorem 9 , we have that $\pi^{*}(1)>\pi^{c}(1)$.

Thus, after finding the value $\beta_{0}$, the optimal socialization level $\widehat{\beta}$ is given by the intersection of the functions

$$
\begin{aligned}
& \pi^{*}(\beta), \\
& \pi^{c}(\beta),
\end{aligned}
$$

within the interval $\left(\beta_{0}, 1\right)$, which, again, we can easily find using a bisection algorithm.

\section{Numerical Experiments and Discussion}

In the classic oligopoly models, the Cournot conjecture, $\partial G / \partial q_{i}=1$, and the perfect competition conjecture, $\partial G / \partial q_{i}=0$, are known to be the extreme cases of the more general conjectural variations conjecture, $\left(\partial G / \partial q_{i}\right)=\omega_{i} \in[0,1]$ (see [14]). In the Cournot equilibrium, the market price is always higher than the market price appearing in the conjectural variations' equilibrium, and compared with the latter, the market price in the perfect competition equilibrium is always lower. Thus, the common perception that the Cournot model is more profitable for the private producers, while the perfect competition model benefits consumers. However, as it was proven in Section 5, this is not always the case when dealing with mixed (or semimixed) oligopolies.

Here, in this section, we provide some numerical experiments to illustrate the different situations (formulated in Theorems 4 to 9) that may arise in the semi-mixed oligopoly and lead to the optimality criterion for the socialization level. The examples are based on the numerical experiments presented in [23] where the electricity energy market was considered.

The active demand is $D=0$ and the inverse demand function is accepted as the following one:

$$
p(G)=50-0.002 G
$$

which means that the demand is given by the following affine function:

$$
G(p)=-50 p+2500
$$

The cost function for the semi-public company is given by the quadratic function

$$
f_{0}\left(q_{0}\right)=0.01 q_{0}^{2}+2 q_{0}
$$

We assume that the number of the private firms is $n=5$ and that all of them have the same quadratic cost function given by the following formula:

$$
f_{i}\left(q_{i}\right) \equiv f(q)=\kappa q^{2}+3.25 q, \quad \forall i \in\{1, \ldots, 5\},
$$

where the value of $\kappa$ is given by $\kappa=0.0075$ for Experiment 1 , $\kappa=0.025$ for Experiment 2, and $\kappa=0.0025$ for Experiment 3 .
In the cost function (51) for the private firms, the parameter $\kappa$ can be interpreted as the strength of the private firms when the production in the market is high (indeed, if the output volume $q$ is large enough, the linear term of the quadratic cost functions is negligible), and thus, for Experiment 1 we consider the private firms to be stronger than the semi-public company, and even more strong for Experiment 3, and for Experiment 2, we consider the private firms to be weaker than the semi-public company.

In all 3 experiments, we compare the equilibriums of consistent conjectural variations (CCVE), Cournot, and perfect competition (P-C). Also, in order not to lose the more intuitive notion of the influence coefficients, we are going to use the following relationships:

$$
\omega_{i} \equiv \frac{\partial G}{\partial q_{i}}=\frac{\partial G}{\partial p} \frac{\partial p}{\partial q_{i}}=-K \frac{\partial p}{\partial q_{i}}=K v_{i}, \quad \forall i \in\{0,1, \ldots, 5\},
$$

to work with the influence coefficients $\omega_{i}$. With this notation, the Cournot conjecture is represented by $\omega_{i}^{c}=1$ for all $i \in\{0,1, \ldots, 5\}$, whereas the perfect competition conjecture is given by $\omega_{i}^{t}=0$ for all $i \in\{0,1, \ldots, 5\}$.

In all the figures, the limit values when $\beta \downarrow 0$ correspond to the classic oligopoly model since the semi-public company focuses solely on profit maximization, and on the other hand, the values corresponding to $\beta=1$ correspond to the mixed oligopoly model where the semi-public company only maximizes social welfare.

Experiment 1. In Figures 1 to 5, we present the graphs of the 3 equilibriums as functions of the socialization level $\beta$.

In the graphs of Figure 1, we see that the consistent influence coefficients lie within the open interval $(0,1)$. This is always true (as one can see from equations (19) and (20), of the consistency criterion, and the relationship (52)), so we can consider the CCVE model as somewhat intermediate between Cournot and perfect competition.

We also can see how the influence coefficients decrease with respect to $\beta$; however, the influence coefficient of the private firms decreases considerably faster compared to the semi-public company, which reflects that, in a mixed oligopoly market, the public firm (even when it is the weakest) has the greatest influence in the market given that it selects its output volume to maximize social welfare, regardless of the production cost.

In Figures 2 and 3, we can see that the semi-public company's production increases in the CCVE and Cournot equilibriums as it leans towards social welfare, which is a consequence of trying to decrease the market price (by increasing the total volume) to maximize social welfare.

As mentioned above, the Cournot conjecture favors the private firms, which is why the increment in the semi-public company's production shown in Figure 2 is considerably larger in the Cournot equilibrium compared with the CCVE equilibrium; thus, maximizing social welfare is especially harder under Cournot competition.

On the other hand, we can see that the private firms' production decreases to compensate for the overproduction 


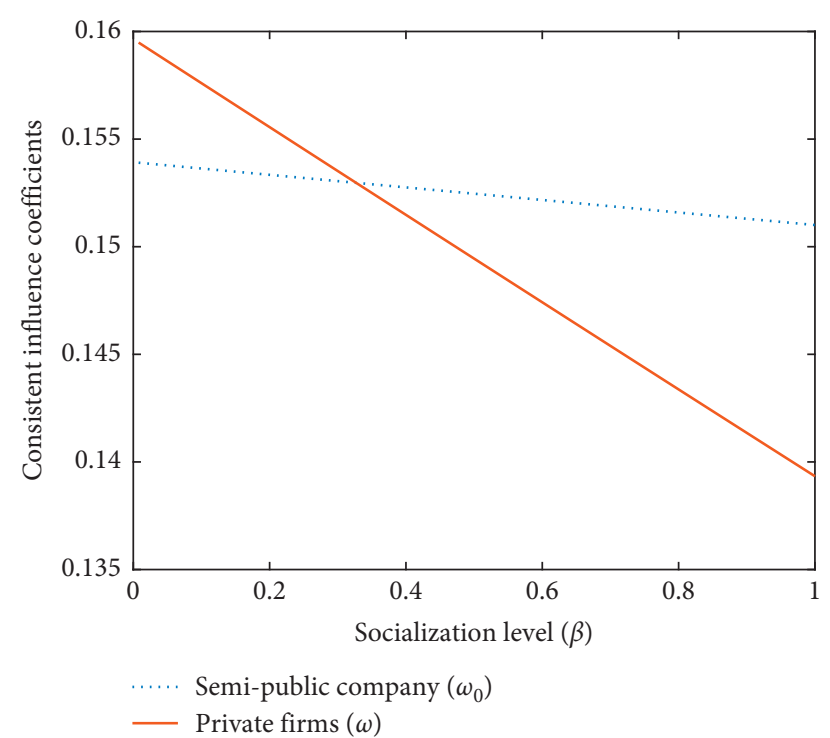

Figure 1: Consistent influence coefficients for the semi-public company and private producers in Experiment 1.

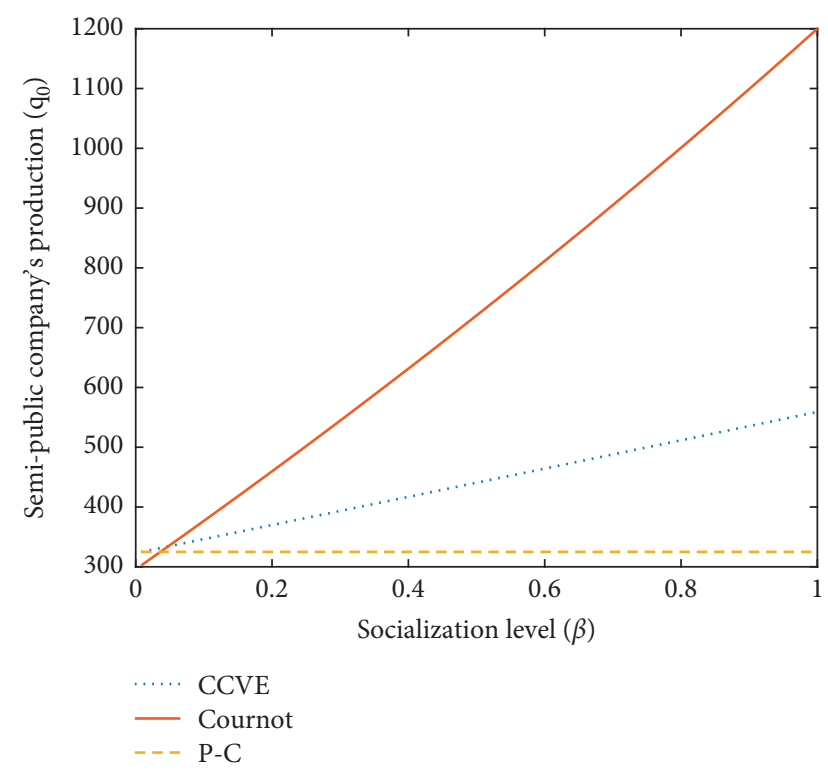

Figure 2: Semi-public company's production for the 3 types of equilibrium in Experiment 1.

of the semi-public company. Meanwhile, for the perfect competition equilibrium, the production output for any producer is always the same regardless of the socialization level $\beta$ chosen by the semi-public company, which reflects the nature of perfect competition (i.e., no producer has influence over the market).

Here, we can see the consequence of a public firm entering a classic oligopoly market; as the public firm seeks to maximize social welfare, the influence of the producers' decreases, approaching perfect competition, thus benefiting the customers; however, in the process, the public firms have to increase its production, forgetting about its profit, which may result in expenses higher than what the public company

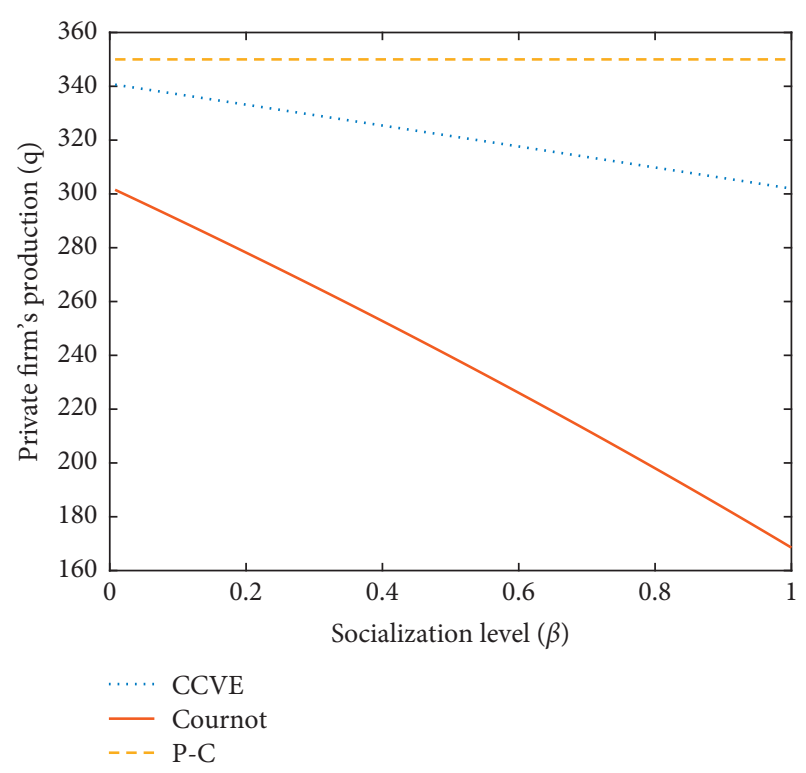

FIgURE 3: Private firms' production for the 3 types of equilibrium in Experiment 1.

can afford. Here is where the importance of the semi-mixed oligopoly framework arises, by maximizing the convex combination of social welfare and net profit, the semi-public company does not only watch over costumers but its own budget too.

In Figure 4, we can see that, in the CCVE and Cournot equilibriums, the market price decreases as the socialization level $\beta$ increases (i.e., as the semi-public company prioritizes social welfare over its net profit), having a greater decrease in the Cournot equilibrium since the market price in the classic oligopoly (i.e., when $\beta \downarrow 0$ ) is considerably higher in the Cournot equilibrium compared to the CCVE equilibrium. Meanwhile, in the perfect competition equilibrium, the market price is constant since the total output volume does not change with respect to $\beta$.

Even so, we can see the intuitive behavior of the market price, the latter being the lowest in the perfect competition equilibrium, the highest in the Cournot equilibrium, and something intermediate in the CCVE equilibrium.

In Figure 5, we can see that, in the classic oligopoly (i.e., when $\beta \downarrow 0$ ), the market follows the intuitive behavior mentioned above, so the Cournot equilibrium shows the highest net profit for the private firms and the perfect competition equilibrium shows the lowest (which again is constant). However, in the mixed oligopoly (i.e., when $\beta=1$ ), the latter is no longer the case as the CCVE equilibrium becomes the most profitable for the private firms whereas the Cournot equilibrium is now the least profitable.

Hence, there exists the socialization level within the open interval $(0,1)$ such that the net profit for the private firms is the same in both, the CCVE and Cournot equilibriums. Thus, by choosing this (optimal) socialization level, the semi-public company can convince the private firms to change its Cournot behavior to that of the CCVE, since their net profit will remain the same; however, the market price will still be lower in the CCVE equilibrium (compared with 


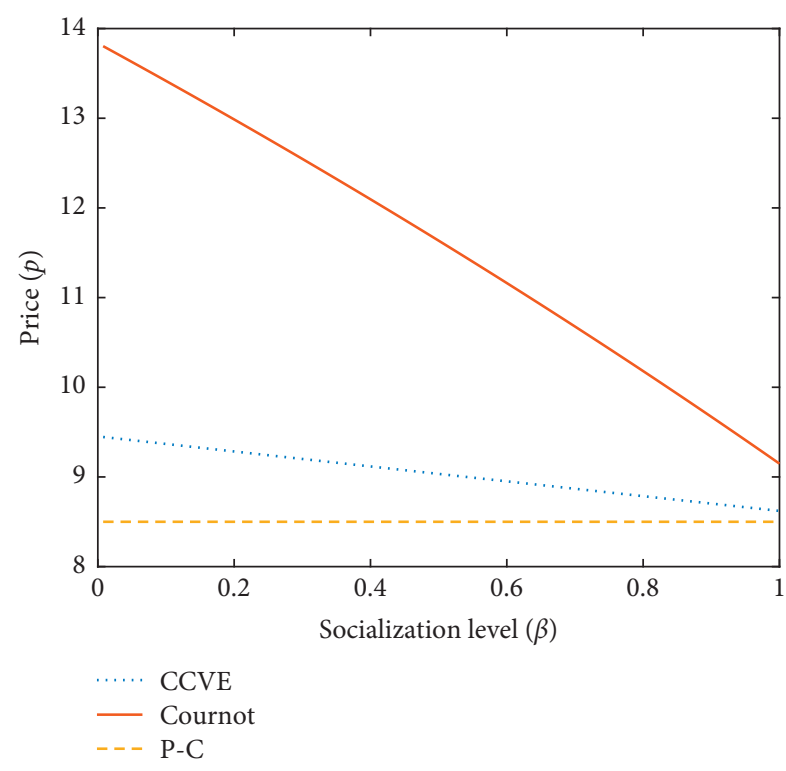

FIGURE 4: Market clearing price for the 3 types of equilibrium in Experiment 1.

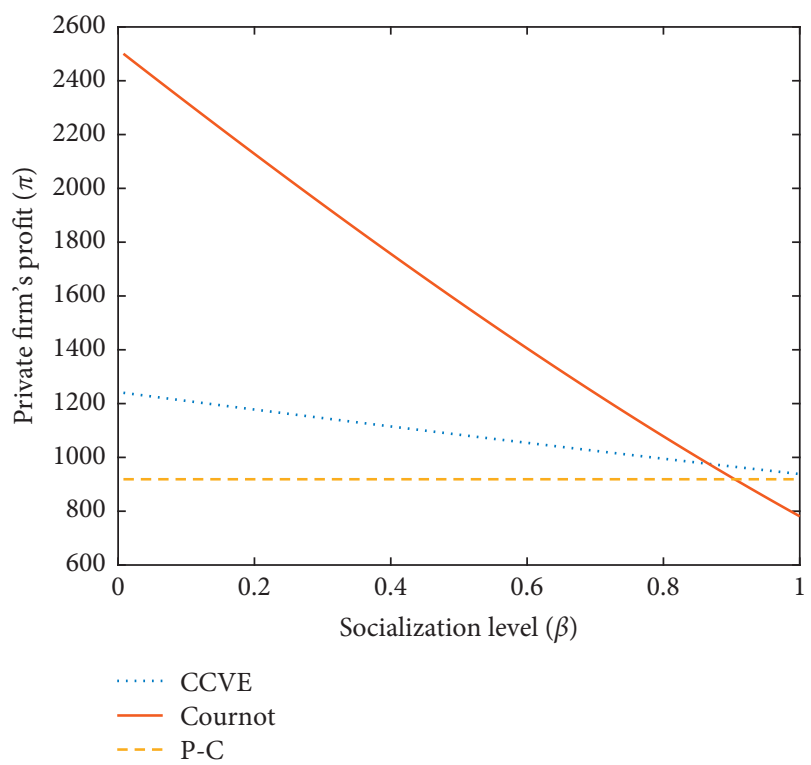

FIGURE 5: Private firms' profit for the 3 types of equilibrium in Experiment 1.

the Cournot equilibrium) so the semi-public company is fulfilling its social responsibility while also taking care of its budget (since the optimal socialization level is less than 1).

For this experiment, the optimal socialization level is $\widehat{\beta} \approx 0.8664$, the corresponding interior equilibrium is

$$
\left(p^{*}, q_{0}^{*}, q^{*}, v_{0}^{*}, v^{*}\right) \approx(8.73,527.37,307.22,0.003028,0.002841),
$$

and the corresponding Cournot (exterior) equilibrium is

$$
\left(p^{c}, q_{0}^{c}, q^{c}\right) \approx(9.84,1065.9,188.39)
$$

having in both equilibriums the private firms' profit of $\pi^{*}=\pi^{c} \approx 975.96$.

Experiment 2. In Figures 6 and 7, we present the market price and private firms' profit functions for the 3 types of equilibrium.

In Figure 6, we can see that the decrease (as $\beta$ increases) in the market price for both, Cournot and CCVE equilibriums, is more notable now that the private firms are weaker, compared to Experiment 1, falling even below the market price in the perfect competition equilibrium. Moreover, in the case of mixed oligopoly (when $\beta=1$ ), the market price of the Cournot equilibrium is now the lowest, which is completely counterintuitive.

In Figure 7, we can see that (as a consequence of the faster decrease in the price) the private firms' net profit in the CCVE and Cournot equilibriums intersects in a smaller socialization level $\beta$, compared to Experiment 1; thus, the semi-public company can convince the private firms to change to the CCVE conjectures, while taking a little more into account its net profit.

Moreover, even though the market price in the Cournot equilibrium becomes the lowest when $\beta=1$, the latter occurs only after the private firms' profit in the CCVE and Cournot equilibriums intersects; therefore, the value of $\beta$ in which this intersection occurs is, again, the optimal socialization level.

For this experiment, the optimal socialization level is $\widehat{\beta} \approx 0.7067$, the corresponding interior equilibrium is

$$
\left(p^{*}, q_{0}^{*}, q^{*}, v_{0}^{*}, v^{*}\right) \approx(14.32,785.52,199.69,0.00713,0.005438),
$$

and the Cournot (exterior) equilibrium is

$$
\left(p^{c}, q_{0}^{c}, q^{c}\right) \approx(14.75,941.52,164.23),
$$

providing in both equilibriums an individual profit for the private firms of $\pi^{*}=\pi^{c} \approx 1213.8$.

Experiment 3. In Figures 8 and 9, we present the market price and private firms' profit functions for the 3 types of equilibrium.

In Figures 8, we see that now (since the private firms are again stronger than the semi-public company) the market price behaves intuitively just like in Experiment 1 . However, since the private firms are now even stronger, the decrease of the market price in the CCVE and Cournot equilibriums is now less notable compared to the decrease in Experiment 1.

In Figure 9, we see that (as a consequence of the market price not decreasing enough) the private firms' net profit in the Cournot equilibrium does not fall below their net profit in the CCVE (nor the perfect competition) equilibrium; thus, the semi-public company (regardless of the socialization level) cannot convince the private firms to change to the CCVE conjectures without paying them any kind of compensation for the losses in their profit. Because of this, there is no optimal socialization level in the sense defined in Section 5 . 


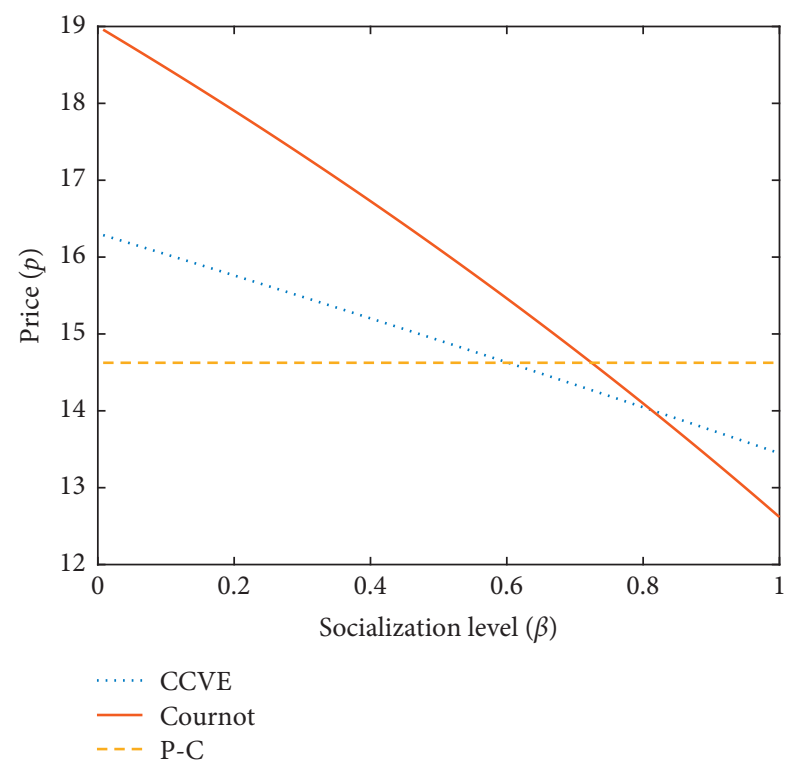

Figure 6: Market clearing price for the 3 types of equilibrium in Experiment 2.

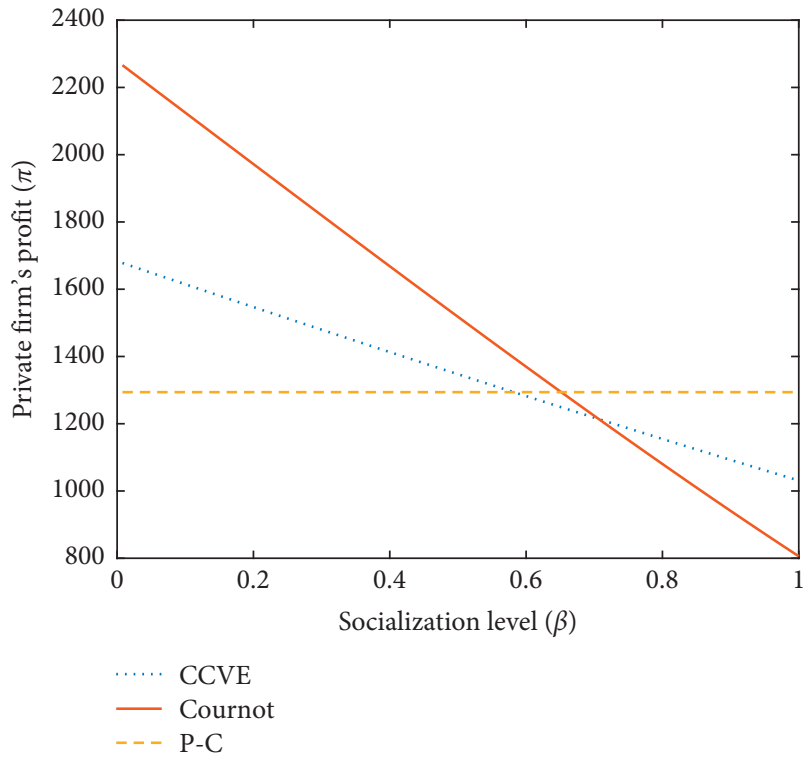

Figure 7: Private firms' profit for the 3 types of equilibrium in Experiment 2.

In [21], the existence of this optimal socialization level was shown for the case of a partially mixed duopoly without the need for relation (41); however, as shown in Experiment 3 , for the case of oligopoly (when the number of producers is at least 3), this optimal socialization level may not exist; in fact, from Theorem 9, we can see that condition (41) is decreasing with respect to both, $a$ and $n$, and thus, we can conclude that the stronger the private firms are (i.e., the smaller the coefficient $a$ is), the larger the number $n$ of private firms should be to guarantee the existence of the optimal socialization level.

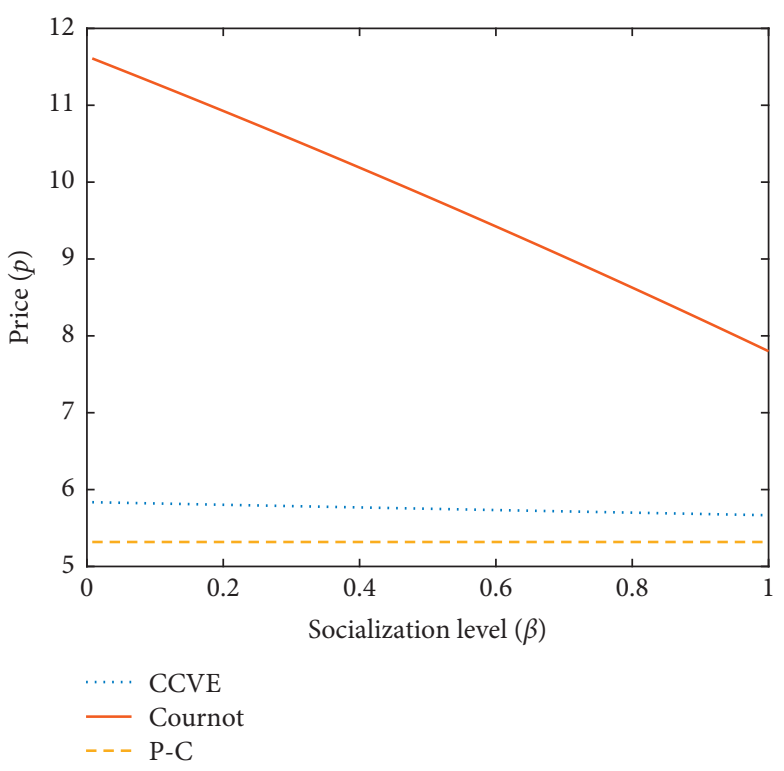

FIGURE 8: Market clearing price for the 3 types of equilibrium in Experiment 3.

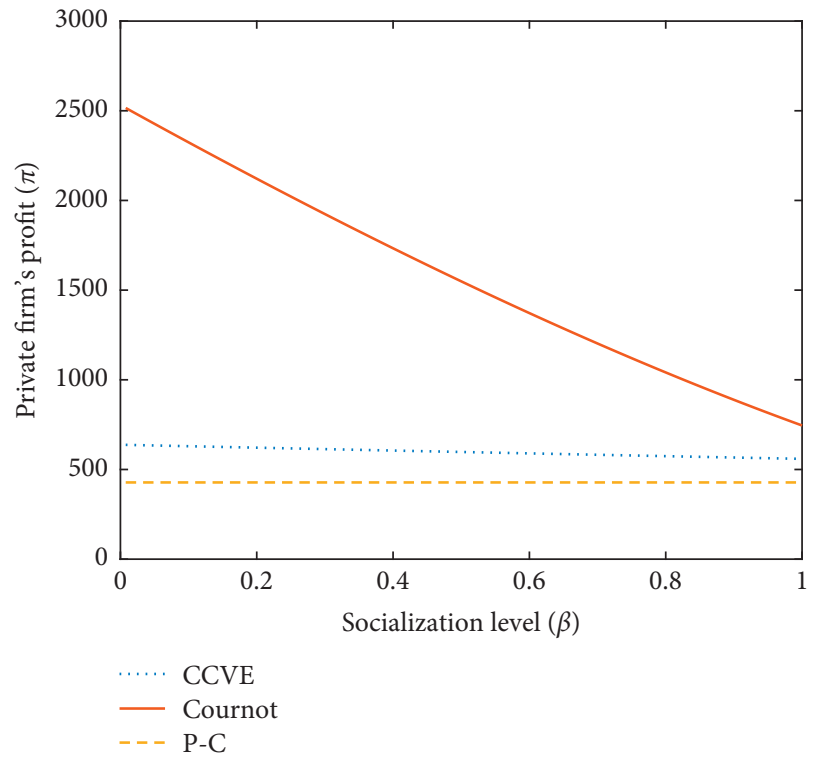

FIgUre 9: Private firms' profit for the 3 types of equilibrium in Experiment 3.

However, even if the optimal socialization level (as defined in Section 5) does not exist, the semi-public company can still convince the private firms to use the CCVE model instead of the Cournot one by paying them a subsidium equal (at the least) to the difference between their expected profits in both models (to make up for their losses).

In our future works, we plan to use this politics of subsidiums to define the optimal socialization level as such that minimizes the total costs of the semi-public company, given as the sum of its production cost and the subsidium that it would be paying to all the private firms. 


\section{Conclusion}

In this paper, we extended the previously studied mixed oligopoly models with conjectural variations equilibrium to the case of the agents' cost functions being convex but not necessarily quadratic. We established the existence and uniqueness results for the conjectural variations equilibrium (called exterior equilibrium) for any set of feasible conjectures. To introduce the notion of interior equilibrium (understood as the CVE with consistent conjectures, or CCVE), we developed a consistency criterion for the conjectures (referred to as influence coefficients) and proved the existence theorem.

Then, we analyzed the behavior of the CCVE, Cournot, and perfect competition equilibriums, and conducted a comparative analysis for the semi-mixed oligopoly with the affine demand function, the firms' cost functions being quadratic, and all private firms having the same cost function. Based on this analysis, we formulated the criterion for the optimal value of the semi-public company's socialization level and proved its existence (under the additional condition that the domestic company cannot be too weak as compared to the private firms). These results were illustrated with numerical experiments for a small electrical power market, showing the different situations that can arise from a public company entering a classic oligopoly market.

In our forthcoming papers, we are going to examine the qualitative behavior of prices and production outputs when the demand function is not necessarily differentiable, and the cost functions are not quadratic.

\section{Data Availability}

No data were used to support this study.

\section{Conflicts of Interest}

The authors declare that they have no conflicts of interest.

\section{Acknowledgments}

This research was financially supported in Mexico by the Secretaría de Educación Pública and the Consejo Nacional de Ciencia y Tecnología (grant number FC-2016-01-1938).

\section{Supplementary Materials}

The PDF file contains the proofs of the lemmas and theorems presented in this work. (Supplementary Materials)

\section{References}

[1] N. Matsushima and T. Matsumura, "Mixed oligopoly and spatial agglomeration," Canadian Journal of Economics, vol. 36, no. 1, pp. 62-87, 2004.

[2] R. C. Cornes and M. Sepahvand, Cournot vs Stackelberg Equilibria with a Public Enterprise and International Competition, University of Nottingham, Nottingham, England, 2003.
[3] C. Fershtman, "The interdependence between ownership status and market structure: the case of privatization," Economica, vol. 57, no. 22, pp. 319-328, 1990.

[4] T. Matsumura, "Stackelberg mixed duopoly with a foreign competitor," Bulletin of Economic Research, vol. 55, no. 3, pp. 275-287, 2003.

[5] T. Matsumura and O. Kanda, "Mixed oligopoly at free entry markets," Journal of Economics, vol. 84, no. 1, pp. 27-48, 2005.

[6] N. J. Ireland and P. J. Law, The Economics of Labour-Managed Enterprises, Croom Helm, London, UK, 1982.

[7] J. P. Bonin and L. G. Putterman, Economics of Cooperation and the Labor-Managed Economy, Harwood Academic Publishers, Geneva, Switzerland, 1987.

[8] F. H. Stephen, The Performance of Labour-Managed Firms, Palgrave Macmillan, London, UK, 1982.

[9] L. Putterman, "Labour-managed firms," in The New Palgrave Dictionary of Economics, S. N. Durlauf and L. E. Blume, Eds., pp. 791-795, Palgrave Macmillan, London, UK, 2008.

[10] B. Saha and R. Sensarma, "State ownership, credit risk and bank competition: a mixed oligopoly approach," Macroeconomics and Finance in Emerging Market Economies, vol. 6, no. 1, pp. 1-13, 2013.

[11] A. Mumcu, S. Oğur, and U. Zenginobuz, Competition between Regulated and Non-regulated Generators on Electric Power Networks, Bogazici University, Istanbul, Turkey, 2001.

[12] A. L. Bowley, "The mathematical groundwork of economics," Social Forces, vol. 3, no. 1, p. 185, 1924.

[13] R. Frisch, "Monopoly, polypoly: the concept of force in the economy," International Economics Papers, vol. 1, pp. 23-36, 1951.

[14] G. Isac, V. A. Bulavsky, and V. V. Kalashnikov, Complementarity, Equilibrium, Efficiency and Economics, Kluwer Academic Publishers, Dordrecht, Netherlands, 2002.

[15] V. A. Bulavsky, "Structure of demand and equilibrium in a model of oligopoly," Economics and Mathematical Methods (Ekonomika I Matematicheskie Metody), vol. 33, pp. 112-134, 1997, in Russian.

[16] V. V. Kalashnikov, V. A. Bulavsky, N. I. Kalashnykova, and F. J. Castillo, "Mixed oligopoly with consistent conjectures," European Journal of Operational Research, vol. 210, no. 3, pp. 729-735, 2011.

[17] N. I. Kalashnykova, V. A. Bulavsky, V. A. Bulavsky, and V. V. Kalashnikov, "Consistent conjectural variations equilibrium in a mixed duopoly," Journal of Advanced Computational Intelligence and Intelligent Informatics, vol. 15, no. 4, pp. 425-432, 2011.

[18] V. V. Pérez, V. A. Bulavsky, V. A. Bulavsky, N. I. Kalashnykova, and J. Watada, "Analysis of consistent equilibria in a mixed duopoly," Journal of Advanced Computational Intelligence and Intelligent Informatics, vol. 18, no. 6, pp. 962-970, 2014.

[19] V. V. Hernández-Rodríguez, V. A. Bulavsky, V. A. Bulavsky, N. I. Kalashnykova, and J. Watada, "Mixed oligopoly: analysis of consistent equilibria," Journal of Advanced Computational Intelligence and Intelligent Informatics, vol. 18, no. 6, pp. 971-984, 2014.

[20] J. G. Flores Muñiz, N. Kalashnykova, V. V. Kalashnikov, and V. Kreinovich, "Public interest and private enterprize: new developments," in Lecture Notes in Networks and Systems, Springer International Publishing, Berlin, Germany, 2021.

[21] V. V. Kalashnikov- Jr., J. G. Flores-Muñiz, V. V. Kalashnikov, and N. I. Kalashnykova, "Consistent conjectural variations equilibrium in a semi-mixed duopoly," Journal of Advanced 
Computational Intelligence and Intelligent Informatics, vol. 21, no. 7, pp. 1125-1134, 2017.

[22] V. A. Bulavsky, "An imagined experiment in the framework of the generalized Cournot model," Economics and Mathematical Methods (Ekonomika I Matematicheskie Metody), vol. 32, pp. 128-137, 1996, in Russian.

[23] Y. Liu, Y. X. Ni, F. F. Wu, and B. Cai, "Existence and uniqueness of consistent conjectural variation equilibrium in electricity markets," International Journal of Electrical Power \& Energy Systems, vol. 29, no. 6, pp. 455-461, 2007. 